\title{
Membrane Stress in the Human Labyrinth and Meniere Disease: A Model Analysis
}

\author{
Daniel J. Pender ${ }^{1}$ \\ ${ }^{1}$ Department of Otolaryngology, Columbia University, New York City, \\ New York, United States \\ Int Arch Otorhinolaryngol 2015;19:336-342.
}

\begin{abstract}
Address for correspondence Daniel J. Pender, MSE, MD, Department of Otolaryngology, Columbia University, 145 West 86th Street, Suite 1C, New York City, NY 10024, United States (e-mail: djp2@columbia. edu).
\end{abstract}

\begin{abstract}
Keywords

- Meniere disease

- labyrinth

- membrane

- stress

- hydrops

Introduction The nature and extent of membrane damage encountered in Meniere disease remains unexplained. Pressure-induced membrane stress may underlie the characteristic hydropic distention. Analysis of stress in the several vestibular chambers may offer insight into the nature and progression of Meniere disease.

Objective Membrane stress levels will be assessed by constructing a specific model of the human membranous labyrinth through the application of human dimensions to an existing generic model of the mammalian labyrinth.

Methods Nominal dimensions for a model of the human membranous labyrinth were obtained from fixed human tissue. Stress proclivities were calculated and normalized based on shell theory applied to the various geometric figures comprising the model. Results Normalized peak stress levels were projected to be highest in the saccule (38.8), followed by the utricle (5.4), then ampulla (2.4), and lowest in the canal system (1.0). These results reflect macrostructural variations in membrane shape, size, and thickness among the several chambers of the labyrinth. These decreasing stress proclivities parallel the decreasing frequency of histologic lesions found in documented cases of Meniere disease.

Conclusions This model analysis of a human membranous labyrinth indicates that substantial disparities in stress exist among the several vestibular chambers due to macrostructural membrane configuration. Low stress levels in the canals are the result of thick highly curved membranes, and the high levels computed for the saccule reflect its thin and relatively flat membranes. These findings suggest that chamber configuration may be a factor controlling the progression of endolymphatic hydrops in Meniere disease.
\end{abstract}

\section{Introduction}

When fluid was first found to fill the bony labyrinth, the delicate membranes suspended therein went undetected. ${ }^{1}$ The use of contrast agents subsequently led to the discovery of the utricle and saccule. ${ }^{2}$ Disparities in the structural thickness of the utricular and saccular membranes were eventually noted. ${ }^{3} \mathrm{~A}$ greater thickness of the utricular membrane was found to be due to a layer of mesothelial connective tissue as well as the presence of dark cells on the epithelial surface of the utricle membrane. ${ }^{4}$ The saccule, lacking such features, was found to be generally thinner but with a localized thickened zone known as Perlman membrane. ${ }^{5}$

Distention of the membranous labyrinth, originally designated hydrops labyrinthi but now better known as endolymphatic hydrops, was found to occur in patients suffering from Meniere disease. ${ }^{6}$ Such membrane distention was found to be nonuniform with the saccule more affected than the utricle. received

February 4, 2015

accepted

February 21, 2015

published online

April 7, 2015
DOI http://dx.doi.org/

10.1055/s-0035-1549157. ISSN 1809-9777.
Copyright $\odot 2015$ by Thieme Publicações License terms Ltda, Rio de Janeiro, Brazil

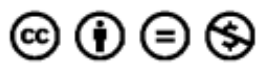


This has been attributed to the saccule's visibly thinner membranes. ${ }^{3}$ Pathologic elevation of endolymphatic fluid pressure has been the suspected cause, supported by the finding of such elevation in experimental hydrops. ${ }^{7}$

Endolymphatic pressure elevation causes distention by inducing tensile stress in the membranes. ${ }^{8}$ In the presence of a static transmural pressure, the tensile stress in the membranes is described by a membrane equation of equilibrium. ${ }^{9,10}$ This membrane equation can be simplified for certain axially symmetrical shapes to permit an assessment of peak stress in these chambers. ${ }^{11}$

A geometric model of the macrostructure of membranous labyrinth in mammals has been described that uses axially symmetric shapes to emulate the several chambers. ${ }^{12}$ In this model, a semicircular canal is treated as a torus, an ampulla as a sphere, the utricle as a cylinder, and the saccule as a discus (i.e., a flattened spheroid). These features are illustrated in - Fig. 1. Stress proclivity (the peak level of stress per unit of transmural pressure) in each chamber of the model has been shown to depend on chamber macrostructure (i.e., shape, size, and thickness of its membranes). ${ }^{13}$

Eq. 1 shows how these entities are related, with stress proclivity given by the product of a shape coefficient and the ratio of chamber radius to membrane thickness.

$$
t / p=C_{s h} \cdot r / w
$$

where $t$ represents tensile stress in the membrane wall; $p$ represents transmural pressure on the membrane; $t / p$ represents stress proclivity; $C_{s h}$ represents a shape coefficient; $r$ represents the axial radius of curvature of the membrane; $w$ represents the wall thickness of the membrane; and $r / w$ represents the membrane thinness index.
Analysis of a model of the human labyrinth may provide insight into the stress disparities inherent to its complex configuration. Such an analysis may also offer a new perspective on the membrane pathology that is characteristic of Meniere disease.

\section{Methods}

A model of the human labyrinth requires specification of chamber proportions and dimensions. Fixed-tissue measurements of the vestibular membranes were used. These measurements were made from a standard temporal bone specimen in the archival collection at Harvard Medical School that was judged to be normal by an independent otopathologist. Nominal measurements of the membranous labyrinth were made with a Zeiss photomicroscope (Germany) fitted with a calibrated reticule. Measurements were taken where the chamber membranes were sectioned transversely. For the external radius of the semicircular canal, a reported value for the horizontal canal was used.

Computations were based on the model chamber geometries of the membranous labyrinth as noted above. ${ }^{12}$ The shape factors for the various geometric configurations used in the model labyrinth have been identified and reported previously. ${ }^{13}$ Model shape coefficients for each chamber were calculated, based on chamber measurements. Nominal dimensions of chamber radii and membrane thickness were utilized to compute a membrane thinness index for each chamber. The greater the ratio of chamber radius to membrane thickness, the "thinner" the membrane is in relative terms.

This value of membrane thinness index was employed to qualify the use of the membrane equation in the determination

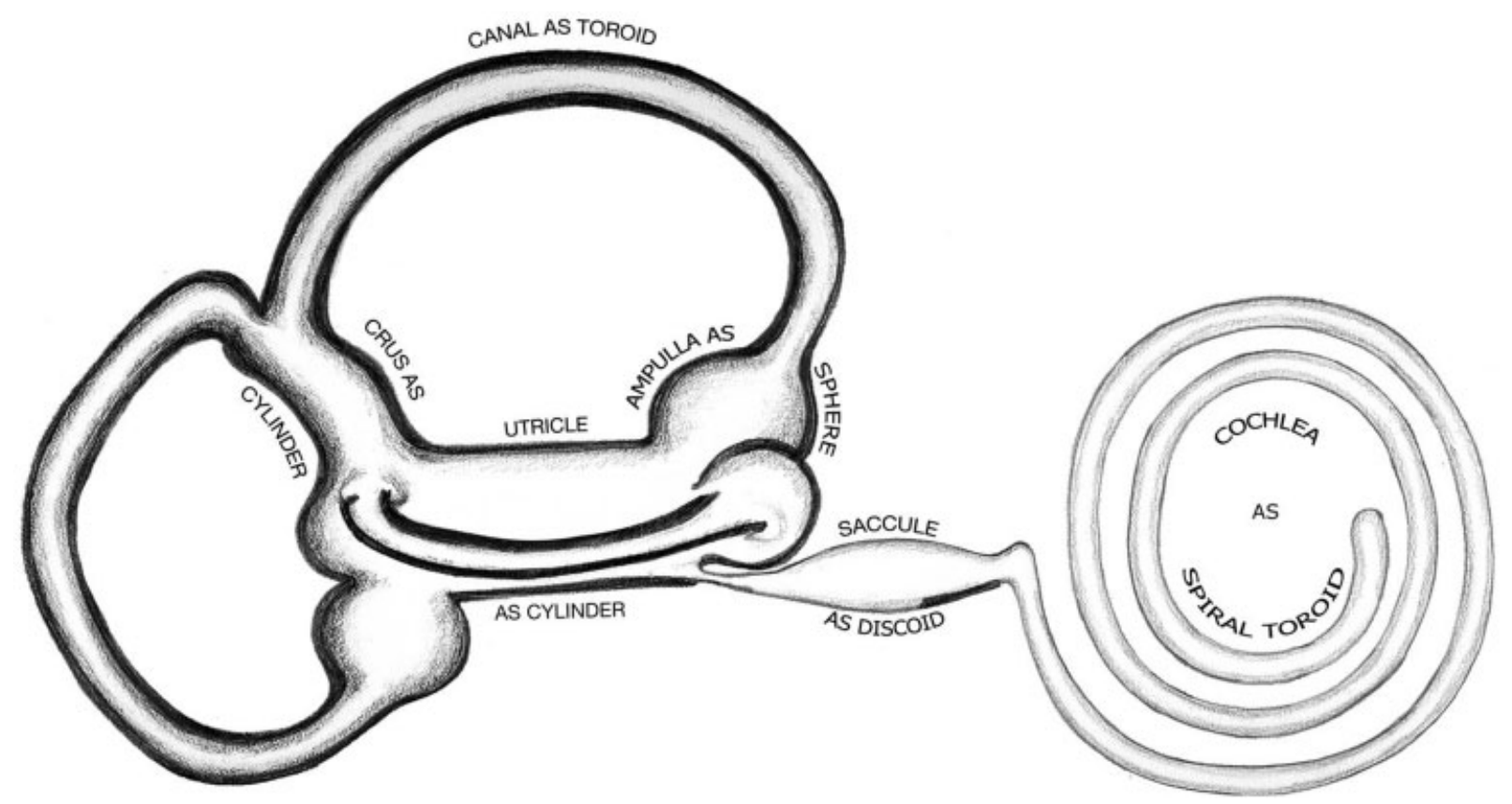

Fig. 1 A model of the human membranous labyrinth using geometrical elements. Heavy lines represent thick membrane in the pars superior and Perlman membrane in the saccule. Light lines represent thin membranes in the saccule and the vestibular membrane (Reissner) of the cochlea spiral. 
of stress proclivity. The membrane equation is only valid for chambers where the membrane thinness index is 5 or larger because the greater the radial size of the chamber for a given membrane thickness, the thinner the membrane relative to chamber size. ${ }^{9}$ Once this qualification was met, stress proclivity was computed as the product of shape coefficient and membrane thinness index using the membrane equation shown in Eq. 1.

Normalized stress in the model was then determined based on the chamber with the least stress proclivity. All measured values were rounded to the nearest whole number in light of their nominal nature, and all calculated values are rounded to the nearest tenth.

\section{Results}

-Table 1 presents the measurements made of the membranous vestibular chambers. Membrane thickness in the range of 23 to $30 \mu \mathrm{m}$, was found to characterize the membranes of the pars superior. In contrast, the saccule demonstrated a much thinner membrane of $\sim 5 \mu \mathrm{m}$, except for its reinforced area with a thickness of $28 \mu \mathrm{m}$. As to the internal radii of curvature of the several chambers, the semicircular canal was found to have a measured value of $140 \mu \mathrm{m}$; the ampulla, a value of $640 \mu \mathrm{m}$; and the utricle, a value of $930 \mu \mathrm{m}$. The lateralmost portion of the saccule had a measured radius of curvature of $1,750 \mu \mathrm{m}$. For the external radius of the model canal, the value of $3,170 \mu \mathrm{m}$ for the horizontal canal as reported by Curthoys and Oman was used in the calculations. $^{14}$

- Table 2 presents a tabulation of results based on these measurements. Membrane shape coefficients ranged from a low of 0.5 for the ampulla modeled as a sphere to a high of 1.1 for the saccule modeled as a discus, with other chamber values intermediate. Membrane thinness index values ranged from a low of 5.6 for the semicircular canal to a high of 204 for the saccule, with intermediate values of 27.8 for the ampulla and 31 for the utricle. All of the computed values for membrane thinness index in - Table $\mathbf{2}$ can be seen to meet the engineering criterion for thin (i.e., the all values are greater than 5.0) and thus validate the use of the membrane equation in their analyses. Of these, the membrane thinness index for the semicircular canal (5.6) makes it effectively the most thick-walled of all the vestibular chambers.

The values for stress proclivities in the model labyrinth were calculated using the membrane shape coefficients and membrane indices in - Table $\mathbf{2}$. Stress proclivity was found to be 5.8 in the semicircular canal, 13.9 in the ampulla, and 31 in the utricle. The stress value for the saccule is estimated to be much higher, with a value of 225 .

Normalized tensile stress proclivity values are also presented in - Table 2. Because the calculations predict that stress proclivity in the semicircular canal will represent the minimum value, normalized stress computations were based on this structure. Therefore, by definition, the value of the normalized stress proclivity in the semicircular canal of the model is 1.0. The normalized stress values for the other vestibular chambers are 2.4 for the ampulla, 5.4 for the utricle, and 38.8 for the saccule. This variation in normalized stress proclivity is shown graphically in -Fig. 2 .

\section{Discussion}

Engineering models have long been used to gain insight into the behavior of unwieldy physical systems. In fact, it has been observed that although "all models are wrong, some are useful." (pg. 424)15 Although modeling is inherently approximate in nature, it can be especially helpful in complex situations where its estimations cannot be readily obtained in any other way. Such a model approach appears well suited to the complex geometry of the membranous labyrinth. Modeling of the individual chambers with simple geometric elements can provide insights that are neither apparent nor intuitive.

The dimensional values used in this model of the human labyrinth are to be considered nominal rather than exact. The measured values for the axial radii of the horizontal semicircular canal, ampulla, and utricle $(140 \mu, 640 \mu, 930 \mu$, respectively) are consistent with the published average norms for these structures $(160 \mu, 580 \mu, 920 \mu$, respectively). ${ }^{14}$ This suggests that the temporal bone utilized in this study was representative of normal and that the

Table 1 Nominal dimensions for a model human labyrinth

\begin{tabular}{|l|l|l|l|l|l|}
\hline Vestibular chamber & Model shape & Wall thickness $(\mu)$ & Axial radius $(\mu)$ & External radius $(\boldsymbol{\mu})$ & Semiaxial length $(\boldsymbol{\mu})$ \\
\hline Canal & Torus & 25 & 140 & $3,170^{\text {b }}$ & n/a \\
\hline Ampulla & Sphere & 23 & 640 & n/a & 640 \\
\hline Utricle & Cylinder & 30 & 930 & n/a & n/a \\
\hline Saccule & Discus & 5 & 1,020 & n/a & 460 \\
\hline Saccule $^{\text {a }}$ & Sphere & 5 & $1,750^{\text {a }}$ & n/a & $n / a$ \\
\hline
\end{tabular}

Abbreviation: n/a, not available.

Note: Values are rounded approximations based on direct measurements of normal human temporal bone tissue, unless noted otherwise. Given the substantial stress disparity between the saccule and the other chambers, an alternate method of calculating peak membrane stress at this point in the saccule was employed. This was based on a direct osculating circle measurement of curvature at the polar region of the saccule ( $1,750 \mu$ ) and the most conservative spherical shape factor (0.5). These data are shown in italics.

${ }^{a}$ Radius of curvature of an osculating circle at the lateralmost portion saccular membrane.

bublished value for the human horizontal semicircular canal. 
Table 2 Calculation of stress parameters in a model human labyrinth

\begin{tabular}{|l|l|l|l|l|l|l|}
\hline $\begin{array}{l}\text { Membrane } \\
\text { structure }\end{array}$ & $\begin{array}{l}\text { Membrane } \\
\text { model }\end{array}$ & $\begin{array}{l}\text { Formula for shape } \\
\text { coefficient }\end{array}$ & $\begin{array}{l}\text { Calculated shape } \\
\text { coefficient }\end{array}$ & $\begin{array}{l}\text { Membrane } \\
\text { thinness index }\end{array}$ & Stress proclivity & $\begin{array}{l}\text { Stress proclivity } \\
\text { normalized }^{\mathbf{a}}\end{array}$ \\
\hline Canal & Torus & $1 / 2(2-r / R) /(1-r / R)$ & 1.03 & 5.6 & 5.8 & 1.0 \\
\hline Ampulla & Sphere & $1-r^{2} / 2 L^{2}$ & 0.5 & 27.8 & 13.9 & 2.4 \\
\hline Utricle & Cylinder & $1-r^{2} / 2 L^{2}$ & 1.0 & 31 & 31 & 5.4 \\
\hline Saccule & Discus & $r / 2 \mathrm{~L}$ & 1.1 & 204 & 225 & 38.8 \\
\hline Saccule & Sphere & $1-r^{2} / 2 L^{2}$ & 0.5 & 350 & 175 & 31.5 \\
\hline
\end{tabular}

Abbreviations: $r$, axial radius; $R$, external radius; $L$, semiaxial length.

Note: Given the substantial stress disparity between the saccule and the other chambers, an alternate method of calculating peak membrane stress at this point in the saccule was employed. This was based on a direct osculating circle measurement of curvature at the polar region of the saccule $(1,750$ $\mu$ ) and the most conservative spherical shape factor (0.5). These data are shown in italics.

${ }^{\mathrm{a}}$ Normalized values are based on the semicircular canal as reference.

supplemental measurements of membrane thickness and curvatures were also within the range of normal.

Earlier work in this area has demonstrated how stress is dependent on chamber configuration. ${ }^{11}$ The tensile stress induced per unit of applied pressure can be very large in a membrane of very low curvature, and conversely, the tensile stress in a highly curved membrane can be quite low, despite be subjected to the same transmural pressure. Indeed, tensile stress can vary from point to point within the membrane of a pressurized vessel of complex configuration, depending on the shape, proportions, thickness, and curvature of the membrane at the point in question. This point is counterintuitive and deserves emphasis. Uniform transmural pressure throughout a structure does not induce a constant level of tension when the containment membranes vary in configuration. Membrane areas with low stress will be more pliant, and others with high stress will be palpably more tense. Thus, the variable geometries of the several chambers in the vestibule suggest that uneven stress vulnerability exists within their membranes.

A quantitative analysis of stress proclivities in the vestibular structures of the toadfish showed that the level of tensile stress induced by transmural pressure does varies consider-

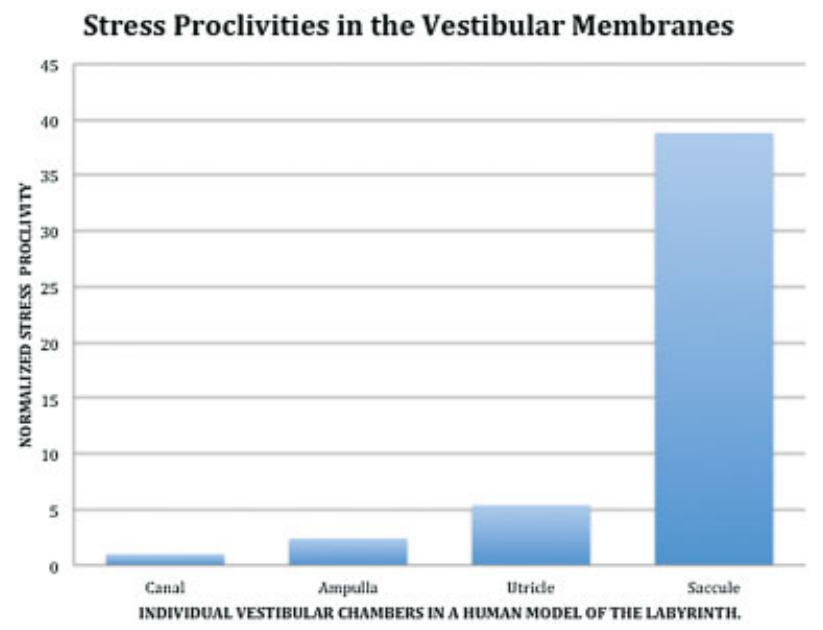

Fig. 2 Disparate stress proclivities in a model of the human labyrinth. The saccule has a stress proclivity that is an order of magnitude greater than the other three chambers. ably with membrane geometry in this species. ${ }^{16}$ Additionally, it has been shown that stress proclivities in the mammalian labyrinth have the potential to reach high levels should extremes of chamber shape be encountered. ${ }^{13}$

The results reported here extend these earlier findings in fish and mammals to the human labyrinth. Suspected disparities in maximum tensile stress in the various chambers are confirmed based on nominal dimensions of a model human labyrinth. Stress levels are projected to be the lowest in the semicircular canal. Stress levels will be 2.4 times higher in the ampulla and 5.4 times higher in the utricle under physiologic pressure in a static equilibrium. However, the most notable finding is that the stress in the saccular membrane is projected to be 38.8 times higher than that in the canal.

Previous work has shown that in the discoid emulation of the saccule, peak stress is meridional and occurs in the polar region, the lateralmost portion of the saccule facing the perilymphatic cistern as shown in - Fig. $\mathbf{3} .{ }^{13}$ Given the substantial stress disparity between the saccule and the other chambers, an alternate method of calculating peak membrane stress at this point in the saccule was employed. This was based on a direct osculating circle measurement of curvature at the polar region of the saccule $(1,750 \mu)$ and the most conservative spherical shape factor $(0.5)$. These data are shown in italics at the bottom of $\boldsymbol{-}$ Tables $\mathbf{1}$ and $\mathbf{2}$. This approach can be considered to establish a conservative lower bound for the actual value because the osculating circle underestimates the flatness of the membrane at the point of measurement. This computation returned a lower bound stress value of 175 , consistent with the model's prediction of 225 .

This high value for stress proclivity does not necessarily mean that the saccule is under extreme tension in the normal state. If the endolymphatic pressure hovers near 0 , then there will be little if any tension in any of the membranes. But whatever tension there is, it will be 38.8 times higher in the saccule than the canal. Furthermore, any rise in pressure beyond the normal physiologic range will be felt more acutely in the saccule. Thus, distention in response to elevated pressure is most likely to affect the saccule primarily. The likelihood of distention in other chambers would be expected 


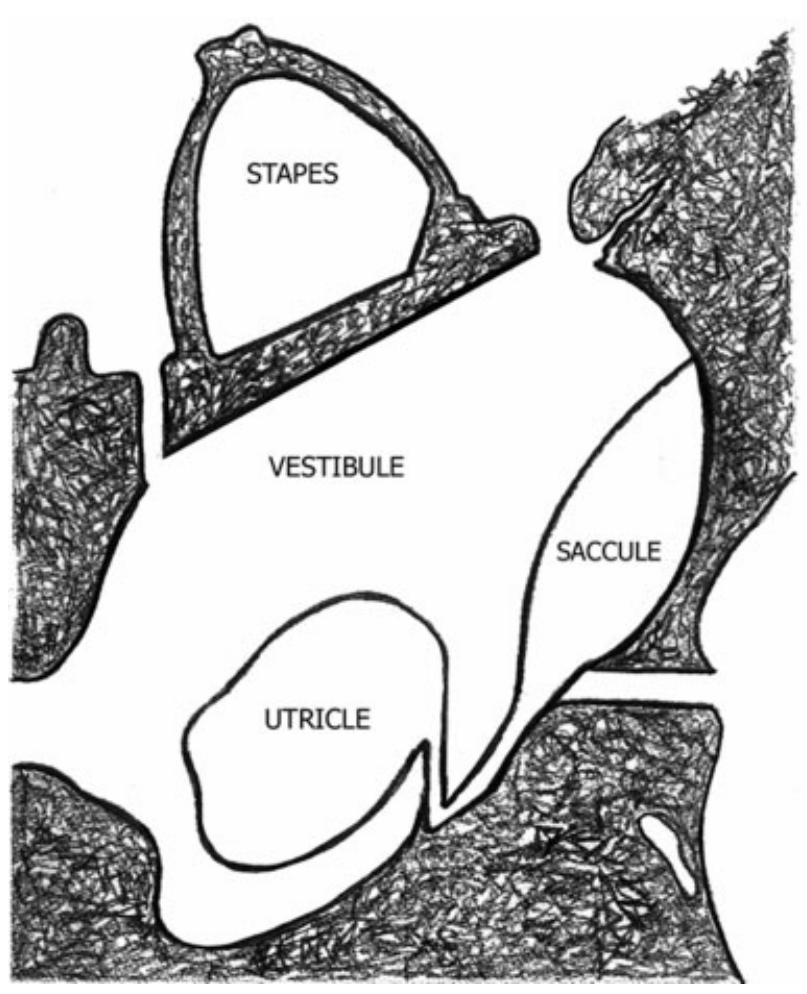

Fig. 3 Schematic of the vestibule showing the key anatomic features. Maximum tensile stress occurs in the center of the saccule membrane.

to parallel their stress proclivities, with utricle second, ampulla third, and canal last.

A better understanding of these disparities comes from closer scrutiny of the controlling parameters of stress in each chamber. The saccule's stress at 225 is much higher than that in the other chambers due to a combination of the adverse flattened shape (discus) and a substantially thinner membrane. Utricular stress at 31 is substantially less due to its smaller radius and thicker membranes. Ampullary stress proclivity at 13.9 is lower still mainly due to its advantageous spherical configuration. The lowest stress proclivity of 5.8 is found in the model canal reflecting its highly curved membranes (low radius of curvature) and relatively thick membrane. It is noteworthy that these features in the canal are the reverse of those encountered in the saccule.

Inspection of the data in - Table $\mathbf{2}$ shows that there is much greater variation in membrane thinness index than there is in shape coefficient values The range of membrane thinness index values ( 5.6 to 204 ) is almost 40 -fold, and that of shape ( 0.5 to 1.1 ) is only twofold. This indicates that the membrane thinness index (radial size relative to membrane thickness) is the dominant determinant of membrane stress; the lower the index, the lower the stress proclivity of the structure.

Comparisons with other bodily structures, namely the vascular and ocular systems, offer some perspective on this. In the vascular system where vessels share a cylindrical shape, large arteries have a membrane thinness index of approximately 4.0 and small arterioles have a value of $1.0 .^{17}$ An arteriole in spasm with virtually no lumen has a membrane thinness index of 0 and is maximally thick in relative terms. Thus, in the distal reaches of the arterial system where the vessels get smaller and smaller, ironically and counterintuitively, the thickness of the vessel walls in relative terms gets larger and larger. In contrast, on the venous side where intravascular pressures are low, the membrane thinness index is $\sim 33$.

In the ocular system, the eye has two spherical components with different curvatures. ${ }^{18}$ The globe has a nominal radius of $12 \mathrm{~mm}$ and a wall thickness of $0.5 \mathrm{~mm}$ at its equator yielding a membrane thinness index $(r / w)$ of 24 . The cornea has a greater curvature with a radius of $8 \mathrm{~mm}$ and central thickness of $0.5 \mathrm{~mm}$, indicating a thin membrane thinness index of 16 . The cornea's lower index indicates a greater mechanical advantage in response to the intraocular pressure and implies lower tensile stress and a greater pliancy than that of the globe.

The membrane thinness index values reported here for the human pars superior range from 5.6 to 31 . These values are broadly consonant with the values for the cardiovascular system (0 to 33 ) and ocular system (16 to 24$)$. It would appear most pars superior structures, namely, utricle, ampullae, and canals, can ordinarily contain endolymphatic pressure with structures that have a membrane thinness index ranging from 5 to $\sim 30$.

However, the saccule's membrane thinness index (204) is at marked variance to those of the older vestibular structures that constitute the pars superior. It reflects the saccule's configuration of low curvature compounded by a thin membrane wall that results in an extraordinarily high membrane thinness index and therefore in relative terms is the thinnest of vestibular membranes. This suggests that the saccule is usually subject only to very low transmural pressure and would be especially vulnerable to any elevation. Theoretically, a thin saccule membrane need not be a liability per se. Were its thin membrane possessed of a high curvature, the computed index could be quite low as with arterioles. However, such is not the case, and the membrane thinness index for the saccule remains remarkably discordant with those of other vestibular structures.

Such disparities in membrane thinness index between saccule and the pars superior structures may be related to evolutionary age and an indicator of relative stability. At one extreme is the semicircular canal with a low index (5.6). This fact would suggest that this structure from a membrane stability point of view would be predicted to be the most stable of all the structures in the vestibular labyrinth. This would be consistent with the evolutionary age of this ancient structure, with its probable origin in the lateral line organ of primitive fish and dating back to the Silurian period some 400 million years ago. ${ }^{19}$ At the other extreme, the saccule of the evolutionarily newer pars inferior has a high membrane thinness index that may indicate instability and potential for distention and rupture, something that can be seen in other structures when membranes are thin and dilated. For example, when a 3-mm cerebral berry aneurism thins out and distends to $10 \mathrm{~mm}$, its theoretical membrane thinness index increases 37 -fold. Such $10-\mathrm{mm}$ aneurisms are attended by a substantial likelihood of rupture. ${ }^{20}$ Thus from the point of view of membrane thinness index, low values would seem to 
be associated with greater membrane stability and high values may portend instability under pressure.

Finally, idealized geometrical figures that have an axis of symmetry are used to model chamber macrostructure in this study. The real vestibular membranes are likely to exhibit departures from these ideal states, and areas of irregularity will entail stress concentrations beyond those projected by the model. In addition, the membranes are presumed to be uniformly thick in the model. This assumption does not take into account real membrane microstructure wherein a composite of elements including collagen and various cellular elements are present and of variable thickness. These features may thus entail peak stress levels beyond those projected by the model. Thus, the model should be construed as providing a conservative estimate of stress proclivities in the various chambers.

These results have certain implications that bear directly on Meniere disease. The lesions in Meniere disease are found to occur in a precise sequence within the membranous labyrinth. ${ }^{21}$ The saccule is the first vestibular chamber to be affected after the cochlea. The utricle, the ampullae, and the canal system are then affected in that exact order. This sequence precisely parallels the order of stress proclivities projected herein for the human vestibular labyrinth, largest in the saccule and least in the canals. This consonance of lesion progression in Meniere disease with the range of stress disparities found in the model is noteworthy and suggests an association between these observations. These findings suggest that chamber macrostructure exerts a moderating influence on the progression of hydrops.

This would appear to be related to the membrane mechanical advantage afforded each chamber by its macrostructural combination of shape, size, and wall thickness. Because hydrops progresses from chamber to chamber in serial fashion following the normal anatomy, chambers in the chain that are mechanically advantaged may be less readily affected by the hydropic process and vice versa. This raises the possibility that chambers in some individuals may be more vulnerable to hydrops if they exhibit macrostructural variations in size, shape, and thickness outside of the normal range that compromises their normal membrane mechanical advantage. Support for this possibility comes from the observation that the saccule and utricle are larger in the contralateral ears of patients with Meniere disease. ${ }^{22}$ Contralateral ears are more likely to develop hydrops than normal ears, ${ }^{23}$ and therefore they may be constitutionally more vulnerable to the hydropic process due to their increased size.

Although membrane stress level depends on macrostructural characteristics of each chamber, chamber distensibility will depend on the microstructure of the membrane. Were the saccule to be made of stainless steel and the canals of gum rubber, it is clear that the canals would distend first under pressure regardless of their macroscopic structure. However, collagen appears to be the membrane constituent that determines distensibility. ${ }^{24}$ Variation in collagen microstructural type, amount, and viscoelastic property can potentially confer nonuniform deformation characteristics on the vestibular chambers. Such membrane microstructure can be expected to modulate the effect of chamber macrostructure in determining overall vestibular response to the hydropic process.

\section{Conclusions}

In this analysis of a model human labyrinth, differences in stress proclivities are projected to exist in the membranes of the several vestibular chambers. This is based on macrostructural variations in chamber size, shape, and membrane thickness. Most affected will be the saccule with its adverse discus shape and its thin membranes. Least affected will be the canal system due to its small radial size and its relatively thick membranes. Utricle and ampullae will be affected to an intermediate extent. These findings parallel the frequent occurrence of hydrops in the saccule and the rare occurrence of hydrops in the canal system, with the utricle and ampullae intermediately affected. Such an association leads to the suspicion that variations in chamber macrostructural geometry due to shape, size, and membrane thickness may be a factor controlling the progression of Meniere disease.

\section{References}

1 Cotugno D. De Aquaeductibus Auris Humanae Internae. Neapoli et Bononiae: Ex Typographia Sancti Thomae Aquinatis; 1775

2 Scarpa A. Anatomische Untersuchungen des Gehors und Geruchs. Nürnberg: Raspe; 1797:76-85

3 Schuknecht HF, Benitez JT, Beekhuis J. Further observations on the pathology of Meniere's disease. Ann Otol Rhinol Laryngol 1962; 71:1039-1053

4 Kimura RS. Distribution, structure, and function of dark cells in the vestibular labyrinth. Ann Otol Rhinol Laryngol 1969;78(3): 542-561

5 Perlman HB. The saccule: observations on a differentiated reenforced area of the saccular wall in man. Arch Otolaryngol 1940; 32:678-691

6 Hallpike CS, Cairns H. Observations on the pathology of Ménière's syndrome: (Section of Otology). Proc R Soc Med 1938;31(11): 1317-1336

7 Ito S, Fisch U, Dillier N, Pollak A. Endolymphatic pressure in experimental hydrops. Arch Otolaryngol Head Neck Surg 1987; 113(8):833-835

8 EFUNDA. Engineering Fundamentals: Thin walled pressure vessels. 2006 Available at: http://www.efunda.com

9 Fung YC. Biomechanics: Mechanical Properties of Living Tissues. New York, NY: Springer-Verlag; 1993

10 Shigley JE, Mischke CR, Budynas RG. Mechanical Engineering Design. New York, NY: McGraw-Hill; 2004

11 Pender DJ. A model analysis of static stress in the vestibular membranes. Theor Biol Med Model 2009;6:19

12 Pender DJ. A model design for the labyrinthine membranes in mammals. Laryngoscope 2014;124(6):E245-E249

13 Pender DJ. Membrane stress proclivities in the mammalian labyrinth. Int Arch Otorhinolaryngol 2014;18:398-402

14 Curthoys IS, Oman CM. Dimensions of the horizontal semicircular duct, ampulla and utricle in the human. Acta Otolaryngol 1987; 103(4):254-261

15 Box GEP. Robustness in the strategy of scientific model building. In: Launer RL, Wilkinson GN, eds. Robustness in Statistics. New York, NY: Academic Press; 1979

16 Pender DJ. A model analysis of tensile stress in the toadfish vestibular membranes. Int J Otolaryngol 2011;2011:519293 
17 Macura KJ, Corl FM, Fishman EK, Bluemke DA. Pathogenesis in acute aortic syndromes: aortic aneurysm leak and rupture and traumatic aortic transection. Am J Roentgenol 2003;181(2): 303-307

18 Shubert HD. Anatomy of the orbit. 2006

19 Kuraku S, Kuratani S. Time scale for cyclostome evolution inferred with a phylogenetic diagnosis of hagfish and lamprey cDNA sequences. Zoolog Sci 2006;23(12):1053-1064

20 Baek S, Rajagopal KR, Humphrey JD. A theoretical model of enlarging intracranial fusiform aneurysms. J Biomech Eng 2006; 128(1):142-149
21 Pender DJ. Endolymphatic hydrops and Ménière's disease: a lesion meta-analysis. J Laryngol Otol 2014;128(10):859-865

22 Morita N, Kariya S, Farajzadeh Deroee A, et al. Membranous labyrinth volumes in normal ears and Ménière disease: a three-dimensional reconstruction study. Laryngoscope 2009;119(11):2216-2220

23 Sperling NM, Paparella MM, Yoon TH, Zelterman D. Symptomatic versus asymptomatic endolymphatic hydrops: a histopathologic comparison. Laryngoscope 1993;103(3):277-285

24 Shinozaki N, Kimura RS. Scanning electron microscopic observations on the distended Reissner's and saccular membranes in the guinea pig. Acta Otolaryngol 1980;90(5-6):370-384 